\title{
A microcomputer package for real-time skin conductance response analysis
}

\author{
JOHN A. SPINKS \\ University of Hong Kong, Hong Kong \\ ROGER DOW \\ Contact Precision Instruments, London, England \\ and \\ LEUNG WAI CHIU \\ University of Hong Kong, Hong Kong
}

\begin{abstract}
A suite of programs has been developed to acquire data from a commercially available, highquality skin conductance meter, via a standard IEEE bus, on ai. Apple II or Apple IIe microcomputer. These programs score and analyze stimulus-evoked skin conductance responses in a preset time window, and store the resultant data on disk, if required. Facilities for a manual override of the automatic scoring routine are available. The setup offers a method of scoring data as they are acquired; it uses currently recommended standards for skin conductance analysis and allows a high magnification of responses, without any need for continual monitoring, because of hardware facilities that automatically "back-off" a subject's tonic skin conductance level.
\end{abstract}

A suite of programs has been developed to access data, via an IEEE bus, from a high-quality meter for measuring skin conductance (SC) activity. The setup conforms to currently accepted standards for SC measurement, scores SC responses (SCRs) immediately following data acquisition, and is portable and easy to use. The equipment and software have been used to run a number of experiments measuring stimulus-evoked SCRs, both with student subjects in an environmentally controlled psychophysiology laboratory and with autistic children as subjects in a psychiatric unit.

\section{INSTRUMENTATION PACKAGE}

SC activity is one of the most widely used physiological indexes of cognitive activity. Proper measurement and scoring, however, is complex (Siddle, Turpin, Spinks, \& Stephenson, 1980) and has not always conformed to the standards agreed upon as being desirable (Venables \& Christie, 1980). The SC meter used in this package is the SC4, developed by Contact Precision Instruments. ${ }^{1}$ The meter is a direct-coupled, constantvoltage device that measures conductance rather than

The authors would like to thank John Bacon-Shone of the University of Hong Kong for his assistance in the development of these facilities. Acknowledgment should also be given to the Committee on Research and Conference Grants of the University of Hong Kong for financial assistance in the research of which this program development forms a part. Please address reprint requests to: John A. Spinks, Department of Psychology, University of Hong Kong, Hong Kong. resistance (Lykken \& Venables, 1971; Venables \& Christie, 1980), and automatically backs-off the subject's tonic SC level. Although some companies are now producing SC modules that conform to Lykken and Venables's specification, it is this automatic back-off feature that is an exciting development in SC recording technology. This is because it allows the user to monitor SC activity to a high degree of resolution, without his or her having to continuously monitor and reset the polygraph, which is necessary with other commercially available SC recorders. There are two alternatives to an automatic back-off system. One is to set the gain so low that the signal does not go off the scale (and thus lose the high degree of resolution available with the present system, even when a 12 -bit $A / D$ converter is used to acquire data from a $\mathrm{SC}$ recorder). Alternatively, the software can have an interrupt facility, with which the acquisition of data can be halted while the operator adjusts the balance control and enters the new values to the computer via the keyboard. The disadvantages of these alternatives are readily apparent. An IEEE interface is available with the SC4, and the Apple II or Apple Ile microcomputer (with IEEE interface) has been used to acquire the data on the IEEE bus. The SC4 meter may also continuously write out to a poly. graph, if visual monitoring is required. Photographs of a typical SCR as presented on the Apple monitor and polygraph, along with the output from another commercially available skin resistance meter, are shown in Figure 1. In a set of SCRs scored by two experienced researchers in psychophysiology, interscorer reliability 


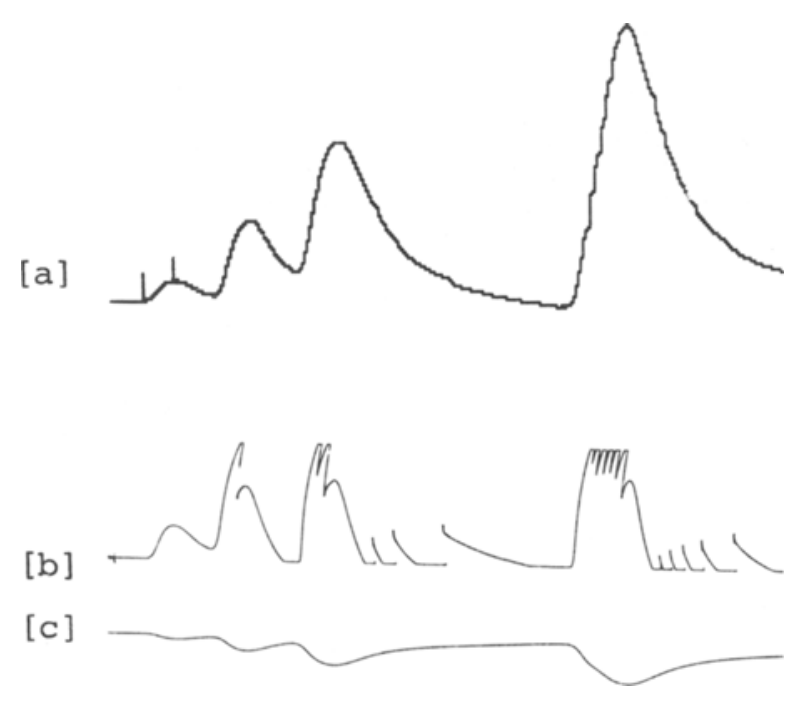

Figure 1. A comparison of graphic output for a sample period of $27.5 \mathrm{sec}$ of electrodermal activity. [a] Output from SC4 meter plotted on Apple monitor. [b] Output from SC4 meter plotted on polygraph. [c] Output from skin resistance meter plotted on polygraph.

was considerably higher for the present microcomputer system (Figure 1a) than for skin resistance responses plotted on the polygraph (Figure 1c). Discrepancies in the first case reached a maximum of $2.97 \%$; in the second case, the maximum was $9.80 \%$.

\section{PROGRAMS}

The programs are written in APPLESOFT BASIC, but may be converted easily to other forms of BASIC. Analysis time windows are determined by the user at the beginning of the program. Data acquisition commences contingent upon a buttonpress on one of the "paddles" (or the open-/closed-apple keys of the Apple IIe) or upon another suitable contact closure (e.g., from a timing device or from another computer). SC is sampled at $10 \mathrm{~Hz}$, and the resultant data are plotted on the monitor screen using the APPLESOFT high-resolution graphics facilities, together with cursors indicating the onset and peak of the SCR. Manual override of the computer's scoring is available, with the second Apple "paddle" being used to move the cursors. The SC cursor levels (in microseimens) are continuously shown on the screen, and the SCR magnitude (change in log microseimens) is shown on the screen when scoring has been completed. The onset latency, response peak latency, onset SCL, and peak SCL may all be stored on disk, enabling the researcher to employ change in log conductance calculation or range-correction techniques (Lykken, 1968), as appropriate, and perform statistical analyses at a later stage. Alternatively, the raw SCL data may be stored on disk for analysis with another program at a later stage. This enables multiple responses to be scored, if desired. This program requires no user intervention once started, but still allows high-resolution SC recording. The program is interactive and selfexplanatory.

\section{SYSTEM REQUIREMENTS}

The basic microcomputer system comprises a $48 \mathrm{~K}$ Apple II Autostart ROM and APPLESOFT in ROM or an Apple IIe with monitor, with one or two 5.25-in. disk drives. An Apple IEEE-488 interface is the only additional card required.

\section{COMMUNICATION PROTOCOL}

The data from the $\mathrm{SC} 4$ is sent along the IEEE bus as a 7-byte string. This string carries details of the SC backoff level, the deviation of the subject's actual SCL from this back-off level, the gain setting of the SC4 meter, and details of particular functions on the meter (e.g., whether the calibration switches are depressed or whether the back-off level has been changed recently). The software triggers the SC4 to send a string of information, reads the information on the IEEE bus, byte by byte, and then decodes it. Reading necessitates "PEEKing" particular memory-mapped input locations, in order to read all 8 bits of each byte, as is necessary with the $\mathrm{SC} 4$ meter.

\section{SOFTWARE AVAILABILITY}

Source listings are available from John A. Spinks, Department of Psychology, University of Hong Kong, Hong Kong. The program must, however, be compiled prior to use. Source and compiled programs are available on floppy diskettes from the authors, but there is a US $\$ 10$ charge for diskette, copying, airmail postage, etc. Requests for copies should specify whether the host computer is an Apple II or an Apple Ile, and whether or not it is limited to only a single disk drive. Publications resulting from the use of this software should cite this source reference.

\section{REFERENCES}

LYKKEN, D. (1968) Neuropsychology and neurophysiology in personality research. In E. F. Borgatta \& W. W. Lambert (Eds.), Handbook of personality theory and research (pp. 413-509). Chicago: Rand McNally.

LYKKen, D., \& Venables, P. H. (1971). Direct measurement of skin conductance: A proposal for standardization. Psychophysiology, 8, 656-672.

Siddee, D. A. T., Tunpin, G., Spinks, J. A., \& Stephenson, D. (1980). Peripheral measures. In H. M. Van Praag, M. H. Lader, O. J. Rafaelsen, E. J. Sachar (Eds.), Handbook of biological 
psychiatry: Part 2. Brain mechanisms and abnormal behaviorPsychophysiology (pp. 45-78). New York: Marcel Dekker.

Venables, P. H., \& Christie, M. J. (1980). Electrodermal activity. In 1. Martin \& P. H. Venables (Eds.), Techniques in psychophysiology (pp. 3-67). Chichester, England: Wiley.

\section{NOTE}

1. Inquiries may be addressed to Contact Precision Instruments at 5-9 Beadon Road, London W6 0EA, England, or at PO Box 951, Fssex. Massachusetts 01929.

(Manuscripl received July 7. 1983 ,

revision accepted tor publataton October 26. 1983.) 